\title{
Grading of pancreatic neuroendocrine neoplasms using pharmacokinetic parameters derived from dynamic contrast-enhanced MRI
}

\author{
WEIWEI ZHAO ${ }^{1 *}$, ZHIYONG QUAN ${ }^{2 *}$, XUFANG HUANG $^{1}$, JING REN $^{1}$, DIDI WEN ${ }^{1}$, \\ GUANGWEN ZHANG $^{1}$, ZHONGQIANG SHI ${ }^{3}$, HONG YIN ${ }^{1}$ and YI HUAN $^{1}$
}

Departments of ${ }^{1}$ Radiology and ${ }^{2}$ Nuclear Medicine, Xijing Hospital, Fourth Military Medical University, Xi'an, Shaanxi 710032; ${ }^{3}$ GE Healthcare Co., Ltd., Shanghai 210000, P.R. China

Received November 26, 2017; Accepted March 19, 2018

DOI: $10.3892 / \mathrm{ol} .2018 .8384$

\begin{abstract}
The present study aimed to evaluate the diagnostic efficacy of pharmacokinetic parameters derived from dynamic contrast-enhanced magnetic resonance imaging (DCE-MRI) in prospective evaluation of pancreatic neuroendocrine neoplasms (pNENs) grading. A total of 25 histologically proven patients with pNENs (30 lesions in total) who underwent DCE-MRI were enrolled. Lesions were divided into G1, G2 neuroendocrine tumor (NET) and G3 NET/neuroendocrine carcinoma (NEC) groups based on their histological findings according to 2017 World Health Organization Neuroendocrine Tumor Classification Guideline. In addition, the same numbers of tumor-free regions were selected using as normal control group. For each group, pharmacokinetic DCE parameters: volume transfer constant $\left(K^{\text {trans }}\right)$; contrast transfer rate constant
\end{abstract}

Correspondence to: Professor Yi Huan, Department of Radiology, Xijing Hospital, Fourth Military Medical University, 169 Changle West Road, Xi'an, Shaanxi 710032, P.R. China

E-mail:weiweidedaolai@163.com

*Contributed equally

Abbreviations: DCE-MRI, dynamic contrast-enhanced magnetic resonance imaging; pNENs, pancreatic neuroendocrine neoplasms; NET, neuroendocrine tumor; NEC, neuroendocrine carcinoma; ROC, receiver operator characteristics; NENs, neuroendocrine neoplasms; PRRT, peptide receptor radionuclide therapy; $K^{\text {trans }}$, volume transfer constant; $k_{\mathrm{ep}}$, contrast transfer rate constant; EES, extravascular extracellular space; $\mathrm{v}_{\mathrm{e}}$, extravascular extracellular space volume fraction; $v_{p}$, plasma volume fraction; TR, repetition time; TE, echo time; FOV, field of view; ROI, region of interest; MRF, Markov random fields; AIF, arterial input function; HPF, high-power field; DWI, diffusion-weighted imaging; ADC, apparent diffusion coefficient

Key words: dynamic contrast-enhanced magnetic resonance imaging, pancreatic neuroendocrine neoplasms, tumor grading, pharmacokinetic parameters, quantitative evaluation $\left(k_{\mathrm{ep}}\right)$; extravascular extracellular space volume fraction $\left(\mathrm{v}_{\mathrm{e}}\right)$; and plasma volume fraction $\left(\mathrm{v}_{\mathrm{p}}\right)$ were calculated with Extended Tofts Linear model. Receiver operator characteristics analysis was conducted to assess the diagnostic efficacy of these parameters in pNENs grading. There were significant differences of $K^{\text {trans }}, k_{\mathrm{ep}}, \mathrm{v}_{\mathrm{e}}$ and $\mathrm{v}_{\mathrm{p}}$ between tumor-free areas and G1, G2 NET ( $\mathrm{P}<0.001)$. The $K^{\text {trans }}$ and $k_{\text {ep }}$ of G1 NET were significantly lower compared with those of $\mathrm{G} 2$ ones $(\mathrm{P}<0.005)$. The area under the curve of $K^{\text {trans }}$ and $k_{\text {ep }}$ in differentiating G2 from G1 NET were 0.767 and 0.846 , respectively. When $K^{\text {trans }}$ was $>0.667$ and $\mathrm{k}_{\text {ep }}>1.644$, the sensitivity of diagnosing G2 NET was the lowest (53.85\%), but the specificity was the highest $(93.75 \%)$. When $K^{\text {trans }}$ was $>0.667$ or $k_{\text {ep }}>1.644$, the sensitivity of diagnosing G2 NET was $92.31 \%$, but the specificity was $75.00 \%$. Pharmacokinetic parameters of DCE-MRI, particularly the quantitative values of $K^{\text {trans }}$ and $k_{\text {ep }}$, are helpful for differentiating G2 NET from G1 ones.

\section{Introduction}

Neuroendocrine neoplasms (NENs) are derived from neuroendocrine cells throughout the human body, and the gastroenteropancreatic tract and lung are two main sites of this disease (1). Pancreatic NENs (pNENs) are a subtype of gastroenteropancreatic NENs (2). pNENs are rare tumors accounting for only $1-2 \%$ of all pancreatic tumors. However, the morbidity has increased substantially in the last four decades (from 1.09 to 5.25 per 100,000 individuals between 1973 and 2004) $(1,3)$. In 2017, the updated WHO classification for pNENs divided NENs into G1, G2, G3 neuroendocrine tumor (NET) and neuroendocrine carcinoma (NEC) based on the histological differentiation, including the Ki-67 proliferation index and the mitotic rate (4). One of the most important aspects to tailor the optimal treatment for the pNENs patients is tumor grading. Patients with well-differentiated pNENs are usually managed with treatment with somatostatin analogues and further treatment such as surgery or peptide receptor radionuclide therapy (PRRT) can be considered $(5,6)$. Patients with poorly differentiated NEC should be referred to the oncology department with no delay (7-9). Although G1 and G2 NET are generally treated as the same entity, there are some differences 
to the treatment strategies of the two in clinical practice. So, an accurate preoperative assessment of grading is a prerequisite for individually tailored lesion therapies and prediction of patient outcomes. The current grading system is based on post-surgery or biopsy pathology, which is time-delayed and invasive. At present, sporadic reports about the preoperative grading of pNENs using $\mathrm{CT}$ and magnetic resonance (MR) can be found (10-12), but they were almost retrospective and based on morphology research. Meanwhile their observation points covered many aspects, including lesion morphology, border, size, bile duct dilatation, vascular invasion, signal intensity, and enhancement ratio, which was multifarious and inconvenient in application.

Dynamic contrast-enhanced MR imaging (DCE-MRI), which allows in vivo imaging of the physiology of the microcirculation, provides information related to the vascularity $(13,14)$. By using appropriate pharmacokinetic model, DCE-MRI can generate a series of quantitative parameters, such as volume transfer constant $\left(K^{\text {trans }}\right)$, contrast transfer rate constant $\left(k_{\mathrm{ep}}\right)$, extravascular extracellular space (EES) volume fraction $\left(\mathrm{v}_{\mathrm{e}}\right)$ and plasma volume fraction $\left(\mathrm{v}_{\mathrm{p}}\right)$. It has been demonstrated that these quantitative parameters can provide valuable information in clinical including characterization of cancers, guidance for treatment planning, early prediction of treatment responses and evaluation of treatment outcomes (15-22). However, to our best knowledge, no study has been done to investigate the DCE-MRI pharmacokinetic parameters and its value in grading of pNENs. Thus, the purpose of this study was to evaluate the quantitative DCE-MRI pharmacokinetic parameters in pNENs and their role in pNENs grading.

\section{Materials and methods}

Patient population. Ethical approval was obtained for this prospective research from the Ethics Committee Board and the informed consent was obtained from all participants before collecting information. From May 2014 to August 2016, 43 patients with suspected pNENs were referred from the Endocrine Department and the Department of Hepatobiliary Surgery in our hospital. For inclusion, the candidates should have documentation of eligibility criteria including: Suspected pNENs by ultrasound, CT or other imaging methods; no any disease influencing pancreas; no contraindications to raceanisodamine hydrochloride injection and MRI examination; and no treatment or intervention to pancreatic mass. Among 43 patients, 18 were excluded due to various reasons. Finally, 25 pNENs patients (30 lesions) confirmed by histopathology were included. The case accrual process was summarized in Fig. 1.

MRI protocol. Prior to scanning, patients were requested to fast at least $4 \mathrm{~h}$. Then, $10 \mathrm{mg}$ anisodamine (Raceanisodamine Hydrochloride Injection; Minsheng Pharmaceutical Co., Hangzhou, China) was injected intramuscularly 10 min before examination. MR images of the pancreases were acquired in our institution on a whole body 3.0 T MR scanner (Discovery MR750; GE Medical Systems, Chicago, IL, USA) with an eight-channel phased-array Torso coil positioned on the superior abdomen. Using variable flip angle T1 mapping, pre-contrast three-dimensional spoiled gradient recalled echo sequence series were performed with flip angles of $3^{\circ}, 6^{\circ}, 9^{\circ}$ and $12^{\circ}$. The other imaging parameters of $\mathrm{T} 1$ mapping were set as follows: Repetition time $(\mathrm{TR})=3.2 \mathrm{msec}$, echo time $(\mathrm{TE})=1.5 \mathrm{msec}$, slices number $=60$, slice thickness $=4 \mathrm{~mm}$, matrix $=260 \times 160$, field of view $(F O V)=360 \times 360 \mathrm{~mm}^{2}$. Then, DCE-MRI scans were performed by a three-dimensional fast spoiled gradient recalled echo sequence with the following parameters: $\mathrm{TR}=3.2 \mathrm{msec}, \mathrm{TE}=1.5 \mathrm{msec}$, flip angle $=12^{\circ}$, $\mathrm{FOV}=360 \times 360 \mathrm{~mm}^{2}$, matrix $=260 \times 160$, slice thickness $=4 \mathrm{~mm}$, slice number $=60$, bandwidth was $83.33 \mathrm{~Hz} /$ pixel. It took $320 \mathrm{sec}$ to complete the DCE-MRI scanning with 40 phases acquired and $8 \mathrm{sec}$ for each phase. Three pre-contrast phases were obtained before bolus injection, then an administration of $0.1 \mathrm{mmol} / \mathrm{kg}$ of Gd-DTPA (Omniscan; GE Healthcare Co., Ltd., Shanghai, China) was performed with a venous cannula at a rate of $2 \mathrm{ml} / \mathrm{sec}$ followed by a $20 \mathrm{ml}$ saline flush.

Data manipulation. Two abdominal radiologists, each with more than 8-year experience in clinical MRI, evaluated the acquired images and determined the placement of regions of interest (ROIs). Another experienced radiologist with more than 20 years of experience reviewed the images and made the decision in consensus when the former 2 observers had differences in reading images.

All the DCE-MRI images were transmitted to a workstation for quantitative analysis using DCE-MRI software package (Omni Kinetics, Version 2.00; GE Healthcare Co., Ltd.). First, The DCE-MRI images were post processed by Markov random fields (MRF) 3D non rigid registration algorithms to correct for patient motion that occurs between acquired phases of the dynamic data due to respiration and other involuntary movements. Second, the individual arterial input function (AIF) was obtained from a ROI in abdominal aorta. Third, identical ROIs were manually drawn on corresponding pancreatic lesions and tumor-free areas respectively. The distance of the two ROIs was at least $2 \mathrm{~cm}$. ROIs were drawn manually over the entire lesion on multiple slices without reaching the perimeter to avoid partial volume effect, necrosis, cystic area and vessel. Finally, Extended Tofts Linear model $(23,24)$ was used to calculate the quantitative parameters: $K^{\text {trans }}, k_{\text {ep }}, \mathrm{v}_{\mathrm{e}}$ and $\mathrm{v}_{\mathrm{p}}$. The mean of each parameter in the ROIs was used for statistical analysis.

Histopathological analysis and grouping. The resected specimens were sent to the Department of Pathology in our hospital for further analysis. All lesions were divided into 3 groups based on $\mathrm{Ki}-67$ proliferation index and mitotic rate according to 2017 WHO Neuroendocrine Tumor Classification Guideline (4). i) G1 NET group: Mitoses Per 10 high-power field (HPF) was <2 and Ki-67 Index was <3\%; ii) G2 NET group: Mitoses Per $10 \mathrm{HPF}$ was 2-20 and/or Ki-67 Index was 3-20\%; and iii) G3 NET/NEC group: Mitoses Per $10 \mathrm{HPF}$ was $>20$ and/or Ki-67 Index was $>20 \%$. Meanwhile, tumor-free areas with the same ROI size but staying away from the lesions at least $2 \mathrm{~cm}$ were selected as tumor-free group (normal control group).

Statistical analysis. All statistical analyses were carried out using SPSS software Version 19.0 (SPSS, Inc., Chicago, IL, 


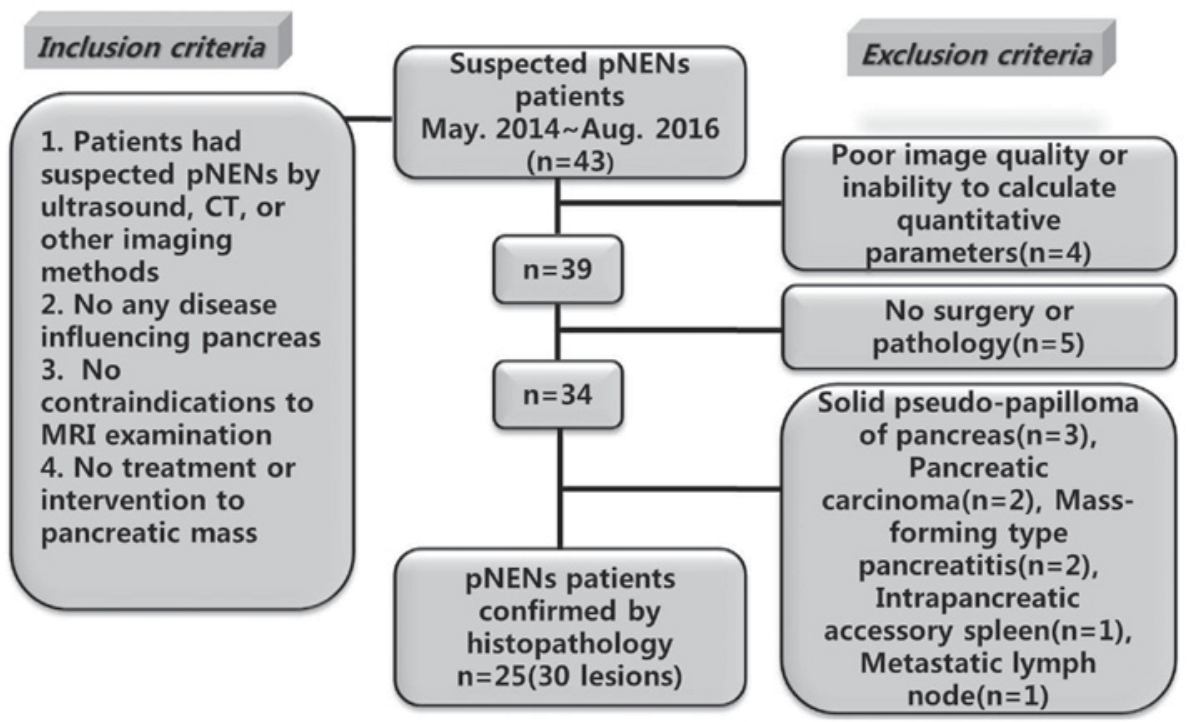

Figure 1. Flowchart showed the study enrollment. Of all the 43 patients, 25 pNENs patients confirmed by histopathology were finally included. pNENs, pancreatic neuroendocrine neoplasms.

USA) and MedCalc software Version 12.3.0.0 (MedCalc Software,. Ostend, Belgium). Data from tumor-free areas, G1 and G2 NET were compared using one-way analysis of variance (ANOVA). Multiple comparison between the groups was performed using LSD method. To find the optimal cut-off levels of DCE parameters to distinguish pNENs grading, the sensitivity and specificity of $K^{\text {trans }}$ and $k_{\text {ep }}$ cut-off values were calculated. Receiver operator characteristics (ROC) analysis was conducted to evaluate the diagnostic ability and assess the appropriate threshold values of $K^{\text {trans }}$ and $k_{\text {ep }} . \mathrm{P}<0.05$ was considered to indicate a statistically significant difference.

\section{Results}

Clinical and pathological characteristics of patients and lesions. The clinical and pathological characteristics of patients and lesions are summarized in Table I. In the final cohort, 25 patients were enrolled in this study (mean age 48.3 years, range $24-68$ years; 11 males with a mean age of 50.9 years and age range of 33-68 years; 14 females with a mean age of 45.7 years and age range of 26-54 years). There were 22 patients with a single lesion, 3 patients with multiple lesions. The total number of lesions were 30 . The maximum in-plane diameter of these lesions ranged from 0.8 to $5.4 \mathrm{~cm}$. The lesions were located in different regions: Head of pancreas $(n=12)$, neck of pancreas $(n=5)$, body of pancreas $(n=7)$, tail of pancreas $(n=6)$. The grades of lesions were as follows: $\mathrm{G} 1$ lesions ( $\mathrm{n}=16), \mathrm{G} 2$ lesions $(\mathrm{n}=13), \mathrm{G} 3$ lesions $(\mathrm{n}=1) . \mathrm{G} 1, \mathrm{G} 2$ and G3 lesions were classified as G1, G2 NET and G3 NET/NEC groups, respectively. According to the 2017 WHO classification of pNENs (4), lesion which is well differentiated morphology, mitotic index $>20$, and/or ki-67 index $>20 \%$ belongs to G3 NET; lesion which is poor differentiated morphology, mitotic index $>20$, and/or ki-67 index $>20 \%$ belongs to NEC. So we set lesions whose mitoses index $>20$ and/or ki-67 index $>20 \%$ into G3 NET/NEC group. There was one lesion in G3 NET/NEC group. In fact, according to the pathology, one well-differentiated G3 NET and zero NEC was in this group.
Comparison of DCE-MRI parameters between pNENs grades. The mean ( $\pm \mathrm{SD}$ ) values of $K^{\text {trans }}, k_{\mathrm{ep}}, \mathrm{v}_{\mathrm{e}}$ and $\mathrm{v}_{\mathrm{p}}$ for tumor-free areas, G1 and G2 NET are presented in Table II. There was only one case in G3 NET/NEC group, so no statistical analysis was performed to this group. Significant differences were found between tumor-free areas and G1, G2 NET regarding $K^{\text {trans }}, k_{\mathrm{ep}}, \mathrm{v}_{\mathrm{e}}$ and $\mathrm{v}_{\mathrm{p}}$ (Table II). The $K^{\text {trans }}, k_{\mathrm{ep}}$ and $\mathrm{v}_{\mathrm{p}}$ of tumor-free areas were significantly lower than those of G1 and G2 NET. However, the $v_{e}$ of tumor-free areas was significantly higher than that of G1 and G2 NET. For the above comparisons, P-values were all less than 0.001 . The $K^{\text {trans }}$ and $k_{\text {ep }}$ of G1 NET were significantly lower than those of $\mathrm{G} 2$ ones $(\mathrm{P}=0.002$ and $\mathrm{P}<0.001$, respectively; Table II and Fig. 2). No significant difference was found between G1 and G2 NET for $v_{e}$ and $v_{p}$ $(\mathrm{P}=0.822$ and $\mathrm{P}=0.419$, respectively). Representative images of two patients with pNENs were showed in Figs. 3 and 4.

Differential diagnostic efficacy of DCE-MRI quantitative parameters in pNENs grading. The diagnostic efficacy of $K^{\text {trans }}$ and $k_{\text {ep }}$ in differentiating G2 from G1 NET are listed in Table III. The ROC curves of $K^{\text {trans }}$ and $k_{\text {ep }}$ are shown in Fig. 5. The AUCs for $K^{\text {trans }}$ and $k_{\mathrm{ep}}$ in differentiating G2 from G1 NET were 0.767 and 0.846 respectively. In the two DCE parameters, $K^{\text {trans }}$ cut-off value of 0.667 provided a specificity of $81.25 \%$; however, the corresponding sensitivity was only $76.92 \%$. The $k_{\text {ep }}$ cut-off value of 1.644 offered moderate diagnostic performance (sensitivity, 69.23\%; specificity, $87.50 \%$ ). When $K^{\text {trans }}$ was over than 0.667 and $k_{\text {ep }}$ exceeded 1.644 , the sensitivity of diagnosing G2 NET was the lowest $(53.85 \%)$, but the specificity was the highest $(93.75 \%)$. When $K^{\text {trans }}$ was over than 0.667 or $k_{\mathrm{ep}}$ exceeded 1.644 , the sensitivity of diagnosing G2 NET was $92.31 \%$, but the specificity is $75.00 \%$.

\section{Discussion}

pNENs are divided into G1, G2, G3 NET and NEC according to the updated 2017 WHO classification of tumor. The 
Table I. Clinical and pathological characteristics of patients and lesions.

\begin{tabular}{lcc}
\hline Characteristic & $\begin{array}{c}\text { No. of } \\
\text { patients }\end{array}$ & $\begin{array}{c}\text { No. of } \\
\text { lesions }\end{array}$ \\
\end{tabular}

\section{Sex}

Male

Female

Single/multiple

Single lesion

Multiple lesions

All lesions

Grading

G1 NET

G2 NET

G3 NET

NEC

13

1

0

Site

Head of pancreas

Neck of pancreas

Body of pancreas

Tail of pancreas

Clinical behaviour

Functional pNENs

Non-functional pNENs

Maximum diameter, $\mathrm{cm}$

$\leq 1$

$>1$ and $\leq 2$

$>2$ and $\leq 4$

$>4$

Heterogeneity

Uniform

Non-uniform

Pattern of enhancement

Fast-in and fast-out

Fast-in and slow-out

Slow-in and slow-out found the benign pNENs were more often round or ovoid in shape than non-benign ones. Main pancreatic duct dilatation was demonstrated only in non-benign pNENs $(\mathrm{P}=0.021)$. In addition, non-benign pNENs had more frequent hypointensity compared with pancreatic parenchyma than benign ones in the arterial phase $(\mathrm{P}=0.029)$. The benign pNENs were significantly smaller than that of the non-benign group $(\mathrm{P}=0.0019)$. The $\mathrm{ADC}$ values of benign pNENs were higher than that of non-benign ones $(\mathrm{P}=0.003)$. Above research were almost retrospective and based on morphology except ADC value. Not surprisingly, above two researchers encountered the same problems as we were in grouping: The number of G3 was too small due to low incidence. So they all set G1 NET as a group, and G2/G3 NET as another group. However, the biological behavior and the treatment strategy are markedly different between G2 and G3 NET, such grouping may not be appropriate. Our original intention was to evaluate the diagnostic efficacy of pharmacokinetic parameters derived from DCE-MRI in prospective evaluation of pNENs grading. Finally, only one G3 NET patient was recruited in the last three years due to the low incidence. So we had to temporarily abandon G3 NET and only analyze the role of pharmacokinetic parameters in distinguishing G1 from G2 ones. As we put in the introduction, there are some differences to the treatment strategies of G1 and G2 pNENs in clinical practice. For example, for the small nonfunctional G1 NET located in pancreatic head, a follow-up can be chosen because of the significant mortality and complications of pancreaticoduodenectomy. However, for G2 NET, which has a higher Ki-67 proliferation index and mitotic rate, the treatment strategy may be aspiring and the follow-up time should be shortened. So it will tailor the optimal treatment for patients with pNENs if G1 and G2 NET could be well classified.

DCE-MRI relies on the use of fast imaging techniques with high temporal resolution and provides quantitative estimation of physiologic parameters related to the microvascular environment in vivo. Recent technical advancements, including parallel imaging and higher magnetic field unit, have enabled us to obtain continuous DCE-MRI images with high temporal resolution of a few sec, which is critical in assessing microvascular circulation. Pharmacokinetic parameters generated from DCE-MRI can help to identify different hemodynamic characteristics and characterize lesions in a quantitative manner. $K^{\text {trans }}$ and $k_{\text {ep }}$ have shown significant differences between G1 and G2 NET in our study. The G2 NET demonstrated a significantly higher $K^{\text {trans }}$ and $k_{\text {ep }}$ than $\mathrm{G} 1$ ones. These findings suggest that DCE-MRI has the potential in differentiating G2 NET from $\mathrm{G} 1$ ones. The absorption and retention of small molecular contrast agent (Gd-DTPA) on tumor mainly depends on blood flow, vascular permeability and the volume of EES (25). $K^{\text {trans }}$ represents the transfer rate of contrast agents from vessels to EES. $k_{\mathrm{ep}}$ represents reflux rate from EES to vessels. Both are related to capillary permeability, meanwhile $K^{\text {trans }}$ also depends on blood flow and capillary surface area. In this study, higher $K^{\text {trans }}$ and $k_{\text {ep }}$ were found in G2 lesions than in G1 ones. Similar phenomenon is also found in other kind of cancers in previous studies. Koo et al (21) found mean $K^{\text {trans }}$ and $k_{\text {ep }}$ were all higher in breast cancers with a higher histologic grade than lower histologic grade. Joo et al (22) found poorly differentiated gastric cancers showed a higher $K^{\text {trans }}$ and $k_{\text {ep }}$ than moderately 
Table II. Comparison of DCE-MRI parameters between different groups.

\begin{tabular}{lcccrr}
\hline Parameter & Tumor-free & G1 NET & G2 NET & P-value $^{\mathrm{a}}$ & P-value $^{\mathrm{b}}$ \\
\hline$K^{\text {trans }}(\mathrm{ml} / \mathrm{min})$ & $0.062 \pm 0.004$ & $0.571 \pm 0.143$ & $0.696 \pm 0.155$ & $<0.001$ & $<0.001$ \\
$k_{\mathrm{ep}}(\mathrm{ml} / \mathrm{min})$ & $0.108 \pm 0.005$ & $1.464 \pm 0.193$ & $1.726 \pm 0.176$ & $<0.001$ & $<0.001$ \\
$\mathrm{v}_{\mathrm{e}}(\mathrm{ml} / \mathrm{ml})$ & $0.604 \pm 0.042$ & $0.411 \pm 0.043$ & $0.408 \pm 0.045$ & $<0.001$ & $<0.001$ \\
$\mathrm{~V}_{\mathrm{p}}(\mathrm{ml} / \mathrm{ml})$ & $0.247 \pm 0.041$ & $0.439 \pm 0.075$ & $0.456 \pm 0.087$ & $<0.001$ & $<0.001$ \\
\hline
\end{tabular}

${ }^{\text {aT }}$ Tumor-free vs. G1 NET; ${ }^{\text {b}}$ Tumor-free vs G2 NET; ${ }^{\mathrm{c}} \mathrm{G} 1$ NET vs. G2 NET. NET, neuroendocrine tumor; $K^{\text {trans }}$, volume transfer constant; $k_{\mathrm{ep}}$, contrast transfer rate constant; $\mathrm{v}_{\mathrm{e}}$, extravascular extracellular space volume fraction; $\mathrm{v}_{\mathrm{p}}$, plasma volume fraction.
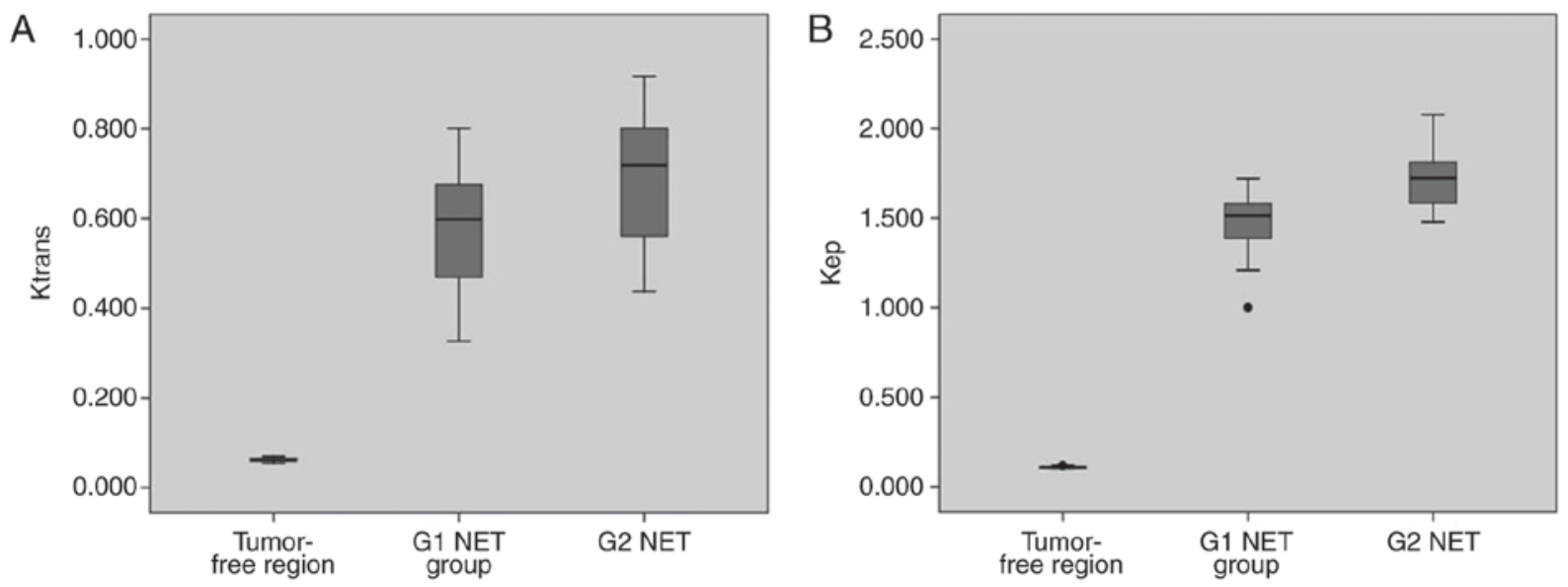

Figure 2. Box plots showing significant difference using ANOVA of (A) $K^{\text {trans }}$ and (B) $k_{\text {ep }}$ among tumor-free areas, G1 and G2 NET. $K^{\text {trans }}$, volume transfer constant; $k_{\mathrm{ep}}$, contrast transfer rate constant; NET, neuroendocrine tumor.
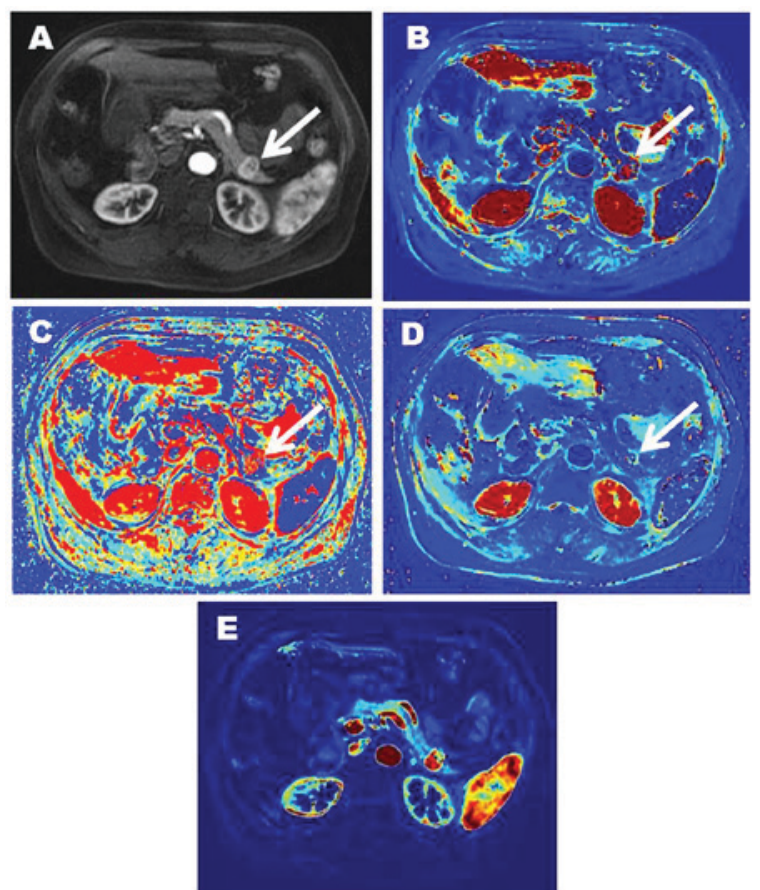

Figure 3. Representative imaging characteristics in a 68-year-old female patient with G1 NET in the tail of pancreas (white arrow). (A; contras enhanced image) shows a markedly enhanced lesion in the tail of pancreas. (B) is $K^{\text {trans }}$ image $\left(K^{\text {trans }}=0.599\right)$. (C) is $k_{\text {ep }}$ image $\left(k_{\mathrm{ep}}=1.441\right)$. (D) is $\mathrm{v}_{\mathrm{e}}$ image $\left(\mathrm{v}_{\mathrm{e}}=0.416\right)$. (E) is $\mathrm{v}_{\mathrm{p}}$ image $\left(\mathrm{v}_{\mathrm{p}}=0.463\right)$. NET, neuroendocrine tumor; $K^{\text {trans }}$, volume transfer constant; $k_{\mathrm{ep}}$, contrast transfer rate constant; $\mathrm{v}_{\mathrm{e}}$, extravascular extracellular space volume fraction; $\mathrm{v}_{\mathrm{p}}$, plasma volume fraction.
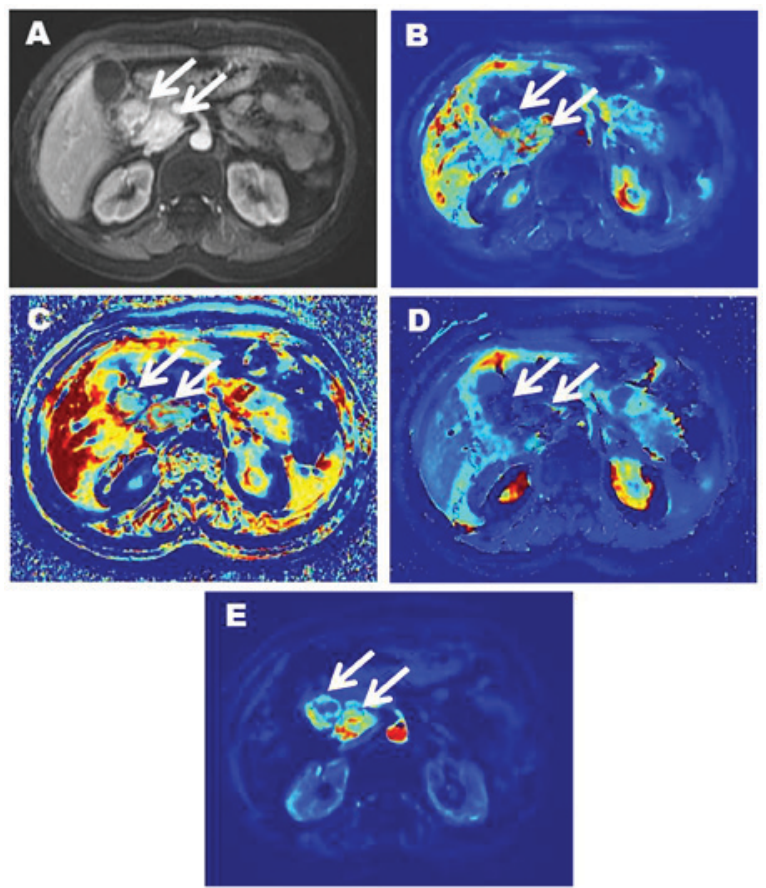

Figure 4. 54-year-old female patient with G2 NET in the head of pancreas (white arrow). (A; contrast enhanced image) shows two significantly enhanced masses in the head of pancreas. (B) is $K^{\text {trans }}$ image $\left(K^{\text {trans }}=0.722\right.$, 0.734).(C) is $k_{\text {ep }}$ image $\left(k_{\mathrm{ep}}=1.783,1.771\right)$. (D) is $\mathrm{v}_{\mathrm{e}}$ image $\left(\mathrm{v}_{\mathrm{e}}=0.406,0.413\right)$. (E) is $\mathrm{v}_{\mathrm{p}}$ image $\left(\mathrm{v}_{\mathrm{p}}=0.438,0.453\right)$. NET, neuroendocrine tumor; $K^{\text {trans }}$, volume transfer constant; $k_{\mathrm{ep}}$, contrast transfer rate constant; $\mathrm{v}_{\mathrm{e}}$, extravascular extracellular space volume fraction; $\mathrm{v}_{\mathrm{p}}$, plasma volume fraction. 
Table III. The diagnostic efficacy of DCE-MRI quantitative parameters in differentiating G2 from G1 NET.

\begin{tabular}{|c|c|c|c|c|c|}
\hline Parameter & Sensitivity & Specificity & PPV & NPV & Accuracy \\
\hline$K^{\text {trans }}$ alone & 0.7692 & 0.8125 & 0.7692 & 0.8125 & 0.7931 \\
\hline$k_{\text {ep }}$ alone & 0.6923 & 0.8750 & 0.8182 & 0.7778 & 0.7931 \\
\hline$K^{\text {trans }}$ or $k_{\text {ep }}$ & 0.9231 & 0.7500 & 0.7500 & 0.9231 & 0.8276 \\
\hline$K^{\text {trans }}$ and $k_{\text {ep }}$ & 0.5385 & 0.9375 & 0.8750 & 0.7143 & 0.7586 \\
\hline
\end{tabular}

NET, neuroendocrine tumor; $K^{\text {trans }}$, volume transfer constant; $k_{\text {ep }}$, contrast transfer rate constant; $K^{\text {trans }}$ alone, $K^{\text {trans }}>0.667 ; k_{\mathrm{ep}}$ alone, $k_{\mathrm{ep}}>1.644$; $K^{\text {trans }}$ or $k_{\text {ep }}, K^{\text {trans }}>0.667$ or $k_{\mathrm{ep}}>1.644 ; K^{\text {trans }}$ and $k_{\text {ep }}, K^{\text {trans }}>0.667$ and $k_{\text {ep }}>1.644$; PPV, positive predictive value; NPV, negative predictive value.

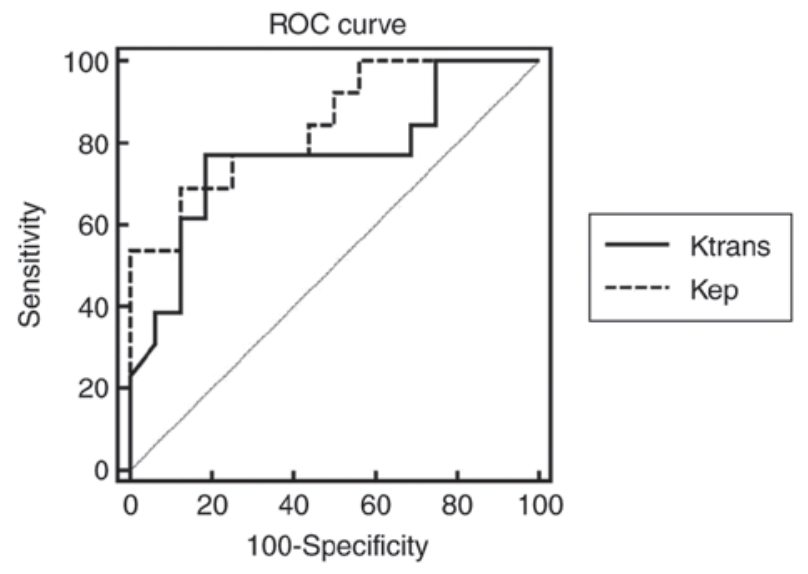

Figure 5. Receiver operator characteristics (ROC) curves for $K^{\text {trans }}$ and $k_{\mathrm{ep}}$ measurements in this study. The solid line represents the ROC curve of $K^{\mathrm{trans}}$, and the dotted line the ROC curve for $k_{\mathrm{ep}}$. The area under the ROC curves (AUCs) for $K^{\text {trans }}$ and $k_{\text {ep }}$ are 0.767 and 0.864 respectively. $K^{\text {trans }}$, volume transfer constant; $k_{\mathrm{ep}}$, contrast transfer rate constant.

differentiated cancers. In most cases, the DCE pharmacokinetic parameters yield composite information about the perfusion and capillary permeability characteristics (13). The uncontrolled angiogenic process requires that new capillaries be recruited from existing blood vessels, in order to ensure a constant supply of nutrients and oxygen, and to allow for the elimination of metabolic waste (26). The increased immature vasculatures contribute to higher perfusion and surface permeability, which result in higher $K^{\text {trans }}$ and $k_{\text {ep. }}$.

$\mathrm{v}_{\mathrm{e}}$ represents the EES volume fraction, approximately equals to the ratio of $K^{\text {trans }}$ to $k_{\mathrm{ep}}$. Previous studies have shown that $\mathrm{v}_{\mathrm{e}}$ increased (22) or decreased (21) with the progression of malignancy. We did not observe higher or lower $\mathrm{v}_{\mathrm{e}}$ in G2 lesions than in G1 ones. This may be due to tumor heterogeneity. $v_{p}$ represents the plasma volume fraction. No statistical difference was found in $v_{p}$ between G1 and G2 NET which may be explained by the immaturity of neovascularization and leaky tumor microcapillary.

In this study, the optimal AUC was achieved by $k_{\mathrm{ep}}$ (AUC $=0.846)$. A sensitivity $(69.23 \%)$ and specificity $(87.50 \%)$ were obtained by adopting a $k_{\text {ep }}$ cut-off value of $1.644 . K^{\text {trans }}$ value of 0.667 offered a moderate sensitivity (76.92\%) and specificity $(81.25 \%)$. When $K^{\text {trans }}$ was over than 0.667 and $k_{\text {ep }}$ exceeded 1.644, the sensitivity of diagnosing G2 pNENs was the lowest $(53.85 \%)$, but the specificity was the highest
(93.75\%). When $K^{\text {trans }}$ was over than 0.667 or $k_{\text {ep }}$ exceeded 1.644 , the sensitivity of diagnosing G2 pNENs was $92.31 \%$, but the specificity is $75.00 \%$. This result is similar, even better than that of previous studies (10-12). However, most of the previous studies are not only based on morphological indicators except for ADC value but also retrospective analysis. In addition, in order to get good differential diagnostic efficacy, previous studies need to combine multiple indicators to analyze. In our research, high sensitivity $(92.31 \%)$ and high specificity $(93.75 \%)$ can be obtained only with appropriate and combined cut-off values of $K^{\text {trans }}, k_{\text {ep }}$. Therefore, $K^{\text {trans }}$ and $k_{\text {ep }}$ may be a potential and ideal screening indicator in the preoperative grading of pNENs.

Small number of patients is a limitation of our study, especially the limited number of patients in G3 NET/NEC group deprived the statistical ability of investigating its correlations between neoplasm grading and DCE-MRI results. This study was a prospective study. Although we were eager, only 25 patients were recruited in the last three years due to the low incidence of pNENs. Fortunately, our study demonstrated the feasibility and potential value of DCE-MRI to differentiate G1 and G2 NET. Further large studies are needed to assess the correlation between DCE-MRI parameters and characteristics of lesions. Therefore, studies are worth to be conducted in larger group of patients, which would further confirm the diagnostic ability of dynamic MR and evaluate cut-off levels depending on the characteristics of patients, lesions and imaging techniques. Our study has additional limitations with regard to the methods of quantitation, including inaccuracies inherent to the manual placement of ROIs. Also, the average values in the ROIs may not reflect the heterogeneous nature of tumor tissue. In the future, larger prospective cohort studies with voxel-based analysis will be required given the relative rarity of pNENs. In addition, another potential limitation which actually not only is a problem of our study but almost of all quantitative precision medicine nowadays needs to be mentioned. DCE-MRI parameters such as $K^{\text {trans }}$ and $k_{\text {ep }}$ could have relatively high variations due to the absence of inter-institutional protocol standardization and inter-vendor differences in hardware/software and may hamper the generalizability of results. Thus, one must take care to use the proposed cut-off values directly in their research unless all procedures are the same with ours described in the paper. In the future, multi-center research and standardization of the procedures are required and would no doubt benefit the generalizability of the results. 
In conclusion, our results have shown the potential value of DCE-MRI in the assessment of pNENs grading. The pharmacokinetic parameters of DCE-MRI, including $K^{\text {trans }}$ and $k_{\text {ep }}$, could provide complementary information in differentiating G2 NET from G1 ones.

\section{Acknowledgements}

Not applicable.

\section{Funding}

This study was funded by the National Natural Science Foundation of China (grant nos. 81370039 and 81220108011).

\section{Availability of data and materials}

The datasets used and/or analyzed during the current study are available from the corresponding author on reasonable request.

\section{Authors' contributions}

YH designed the research, applied for funding and advanced the progress of the research. WZ, ZQ and JR collected, analyzed and interpreted the patient data. WZ and ZQ were major contributors in writing the manuscript. XH, DW and GZ performed the appointments and scanning of the subjects. ZS provided technical support regarding the image post-processing. YH and HY supervised the research group. In addition, HY played a role in the design of the research and the interpretation of the results.

\section{Ethics approval and consent to participate}

Ethical approval was obtained for this prospective research from the Ethics Committee Board of Xijing Hospital (Xi'an, China) and written informed consent was obtained from all participants before collecting information.

\section{Consent for publication}

Written informed consent was obtained from all participants and they consented to publication of their images.

\section{Competing interests}

The authors declare that they have no competing interests.

\section{References}

1. Yao JC, Hassan M, Phan A, Dagohoy C, Leary C, Mares JE, Abdalla EK, Fleming JB, Vauthey JN, Rashid A and Evans DB: One hundred years after 'carcinoid': Epidemiology of and prognostic factors for neuroendocrine tumors in 35,825 cases in the United States. J Clin Oncol 26: 3063-3072, 2008.

2. Dong M, Phan AT and Yao JC: New strategies for advanced neuroendocrine tumors in the era of targeted therapy. Clin Cancer Res 18: 1830-1836, 2012.

3. Halfdanarson TR, Rabe KG, Rubin J and Petersen GM: Pancreatic neuroendocrine tumors (PNETs): Incidence, prognosis and recent trend toward improved survival. Ann Oncol 19: 1727-1733, 2008.
4. Kim JY, Hong SM and Ro JY: Recent updates on grading and classification of neuroendocrine tumors. Ann Diagn Pathol 29: 11-16, 2017.

5. Boudreaux JP, Klimstra DS, Hassan MM, Woltering EA, Jensen RT, Goldsmith SJ, Nutting C, Bushnell DL, Caplin ME and Yao JC; North American Neuroendocrine Tumor Society (NANETS): The NANETS consensus guideline for the diagnosis and management of neuroendocrine tumors: Well-differentiated neuroendocrine tumors of the jejunum, ileum, appendix, and cecum. Pancreas 39: 753-766, 2010.

6. Crippa S, Partelli S, Bassi C, Berardi R, Capelli P, Scarpa A, Zamboni $\mathrm{G}$ and Falconi M: Long-term outcomes and prognostic factors in neuroendocrine carcinomas of the pancreas: Morphology matters. Surgery 159: 862-871, 2016.

7. Crippa S, Partelli S, Belfiori G, Palucci M, Muffatti F, Adamenko O, Cardinali L, Doglioni C, Zamboni G and Falconi M: Management of neuroendocrine carcinomas of the pancreas (WHO G3): A tailored approach between proliferation and morphology. World J Gastroenterol 22: 9944-9953, 2016.

8. Sorbye H, Strosberg J, Baudin E, Klimstra DS and Yao JC: Gastroenteropancreatic high-grade neuroendocrine carcinoma. Cancer 120: 2814-2823, 2014.

9. Kunz PL, Reidy-Lagunes D, Anthony LB, Bertino EM, Brendtro K, Chan JA, Chen H, Jensen RT, Kim MK, Klimstra DS, et al: Consensus guidelines for the management and treatment of neuroendocrine tumors. Pancreas 42: 557-577, 2013.

10. Kim DW, Kim HJ, Kim KW, Byun JH, Song KB, Kim JH and Hong SM: Neuroendocrine neoplasms of the pancreas at dynamic enhanced CT: Comparison between grade 3 neuroendocrine carcinoma and grade 1/2 neuroendocrine tumour. Eur Radiol 25: $1375-1383,2015$.

11. Kim JH, Eun HW, Kim YJ, Han JK and Choi BI: Staging accuracy of $\mathrm{MR}$ for pancreatic neuroendocrine tumor and imaging findings according to the tumor grade. Abdom Imaging 38: 1106-1114, 2013.

12. Jang KM, Kim SH, Lee SJ and Choi D: The value of gadoxetic acid-enhanced and diffusion-weighted MRI for prediction of grading of pancreatic neuroendocrine tumors. Acta Radiol 55: 140-148, 2014

13. Tofts PS, Brix G, Buckley DL, Evelhoch JL, Henderson E, Knopp MV, Larsson HB, Lee TY, Mayr NA, Parker GJ, et al: Estimating kinetic parameters from dynamic contrast-enhanced T(1)-weighted MRI of a diffusable tracer: Standardized quantities and symbols. J Magn Reson Imaging 10: 223-232, 1999.

14. Morgan B, Thomas AL, Drevs J, Hennig J, Buchert M, Jivan A, Horsfield MA, Mross K, Ball HA, Lee L, et al: Dynamic contrast-enhanced magnetic resonance imaging as a biomarker for the pharmacological response of PTK787/ZK 222584, an inhibitor of the vascular endothelial growth factor receptor tyrosine kinases, in patients with advanced colorectal cancer and liver metastases: Results from two phase I studies. J Clin Oncol 21: 3955-3964, 2003

15. Cho E, Chung DJ, Yeo DM, Sohn D, Son Y, Kim T and Hahn ST: Optimal cut-off value of perfusion parameters for diagnosing prostate cancer and for assessing aggressiveness associated with Gleason score. Clin Imaging 39: 834-840, 2015.

16. Park JJ, Kim CK, Park SY, Simonetti AW, Kim E, Park BK and Huh SJ: Assessment of early response to concurrent chemoradiotherapy in cervical cancer: Value of diffusion-weighted and dynamic contrast-enhanced MR imaging. Magn Reson Imaging 32: 993-1000, 2014.

17. Chakiba C, Cornelis F, Descat E, Gross-Goupil M, Sargos P, Roubaud G and Houédé N: Dynamic contrast enhanced MRI-derived parameters are potential biomarkers of therapeutic response in bladder carcinoma. Eur J Radiol 84: 1023-1028, 2015.

18. Furukawa M, Parvathaneni U, Maravilla K, Richards TL and Anzai Y: Dynamic contrast-enhanced MR perfusion imaging of head and neck tumors at 3 Tesla. Head Neck 35: 923-929, 2013.

19. Huang B, Wong CS, Whitcher B, Kwong DL, Lai V, Chan Q and Khong PL: Dynamic contrast-enhanced magnetic resonance imaging for characterising nasopharyngeal carcinoma: Comparison of semiquantitative and quantitative parameters and correlation with tumour stage. Eur Radiol 23: 1495-1502, 2013.

20. Yuan Y, Kuai YP, Chen XS and Tao XF: Assessment of dynamic contrast-enhanced magnetic resonance imaging in the differentiation of malignant from benign orbital masses. Eur J Radiol 82: 1506-1511, 2013 
21. Koo HR, Cho N, Song IC, Kim H, Chang JM, Yi A, Yun BL and Moon WK: Correlation of perfusion parameters on dynamic contrast-enhanced MRI with prognostic factors and subtypes of breast cancers. J Magn Reson Imaging 36: 145-151, 2012.

22. Joo I, Lee JM, Han JK, Yang HK, Lee HJ and Choi BI: Dynamic contrast-enhanced MRI of gastric cancer: Correlation of the perfusion parameters with pathological prognostic factors. J Magn Reson Imaging 41: 1608-1614, 2015.

23. Filice $\mathrm{S}$ and Crisi G: Dynamic contrast-enhanced perfusion MRI of high grade brain gliomas obtained with arterial or venous waveform input function. J Neuroimaging 26: 124-129, 2016.

24. Handayani A, Triadyaksa P, Dijkstra H, Pelgrim GL, van Ooijen PM, Prakken NH, Schoepf UJ, Oudkerk M, Vliegenthart R and Sijens PE: Intermodel agreement of myocardial blood flow estimation from stress-rest myocardial perfusion magnetic resonance imaging in patients with coronary artery disease. Invest Radiol 50: 275-282, 2015.
25. Jennings $\mathrm{D}$, Raghunand $\mathrm{N}$ and Gillies RJ: Imaging hemodynamics. Cancer Metastasis Rev 27: 589-613, 2008.

26. Turkbey B, Kobayashi H, Ogawa M, Bernardo M and Choyke PL: Imaging of tumor angiogenesis: Functional or targeted? AJR Am J Roentgenol 193: 304-313, 2009.

(i) (9) This work is licensed under a Creative Commons Attribution-NonCommercial-NoDerivatives 4.0 International (CC BY-NC-ND 4.0) License. 